\title{
Parametric modelling of a reconfigurable wave energy device
}

\author{
L. Papillon $^{\mathrm{a}, *}$, L. Wang ${ }^{\mathrm{a}}, \mathrm{N}$. Tom ${ }^{\mathrm{b}}$, J. Weber ${ }^{\mathrm{b}}$, J. Ringwood ${ }^{\mathrm{a}}$ \\ ${ }^{\text {a }}$ Centre for Ocean Energy Research, Maynooth University, Co. Kildare, Ireland \\ ${ }^{\mathrm{b}}$ National Renewable Energy Laboratory, MS 3811, 15013 Denver West Parkway, Golden, CO 80401, USA
}

\section{A R T I C L E I N F O}

\section{Keywords:}

Oscillating surge wave energy converter

Parametric modelling

Geometry control

Power absorption control

Hydrodynamic gain

Wind/wave energy comparison

\begin{abstract}
A B S T R A C T
To date, one of the difficulties with wave energy devices is modulating the power transfer from the waves to the generator. In wind turbines, this is achieved by turbine pitch control, leading to the ubiquitous flat power curve. Recently, a wave energy converter, having moveable vanes, has been developed by the National Renewable Energy Laboratory (NREL) in the US. This paper described the development of a compact hydrodynamic model for the NREL device, based on multiple linear hydrodynamic modelling (Murray-Smith and Johansen, 1997), which can be used to model the hydrodynamic behaviour of the device across its full operational spectrum of vane angles. To show the utility of the model, we demonstrate a flat power curve for the WEC, using the vane angles as a control input.
\end{abstract}

\section{Introduction}

Wave energy converters (WECs) are designed to capture the energy carried by ocean waves. Different concepts have been developed in past decades, as reviewed in (Falcão and Apr 2010). Among them, a promising type is a reconfigurable WEC that the geometry of the primary capture system can be controlled. One representative device is the WEC recently developed by National Renewable Energy Laboratory (NREL) in the US, see (Tom et al., 2016a). This reconfigurable WEC enables the adaption of the hydrodynamic gain, which can be thought of as the excitation force kernel gain between the wave power and the excitation torque experienced by the WEC, as shown in Fig. 1. One benefit of adapting the hydrodynamic gain is to modulate the wave load on the device, in particular under high-power or extreme waves, yielding better survivability capacity of the WEC in extreme working environments. Another benefit is that the level of the wave power captured by the device can be tuned, which allows for the approach of a nominal absorbed power of the WEC, combined with wave-to-wave control of the power-take-off (PTO) system. These benefits are vital to the WEC commercial operability in real seas, and can effectively extend the operating envelope of the WEC.

A somewhat similar control strategy has been widely investigated for wind turbines, and comparisons with wave energy are made in (Ringwood and Simani, 2015). As shown in the 2D power curve scheme in Fig. 2, at high wind speeds between rated output wind speed and cut-out speed, output power of the wind energy converter is kept constant, i.e. the rated power, and this is typically approached by adjusting the pitch angle of wind turbine blades (see (Ringwood and Simani, 2015), Fig. 2). This principle can be applied to configurable WECs: the hydrodynamic gain is increased to improve the power production when the wave power drops into a range between cut-in value and rated value, and is reduced to approach the rated power when wave power is higher than the rated value and lower than the cut-out value. The system of controlling the hydrodynamic gain needs to be integrated into an overall control system, e.g. the hierarchical control system shown in Fig. 3. More information on this control strategy can be found in (Ringwood et al., 2014). Based on the information regarding the power carried by incident waves and information of load, the hierarchical control system calculates the optimal setpoint and coordinates the low-level control systems of hydrodynamic gain, generator and power electronic converters. Mechanical or electrical mechanisms can be employed for tuning the hydrodynamics gain, operated on a sea-state-to-sea-state time scale, while the electrical control on the power take-off (PTO) system performs on a wave-to-wave time scale and the power electronics works much faster, regarding to grid fluctuations.

The present article is focused on hydrodynamic gain control, in particular the associated parametric modelling and its application in calculation of absorbed power, using multi-linear hydrodynamic modelling (Murray-Smith and Johansen, 1997). Multi-linear models for flap-type WECs have been reported elsewhere, for example in (Crooks, 2017) and (McCabe et al., 2006). However, these researchers have focused on the use of multi-linear models to model the hydrodynamic

\footnotetext{
* Corresponding author.

E-mail address: louis.papillon67@gmail.com (L. Papillon).
} 


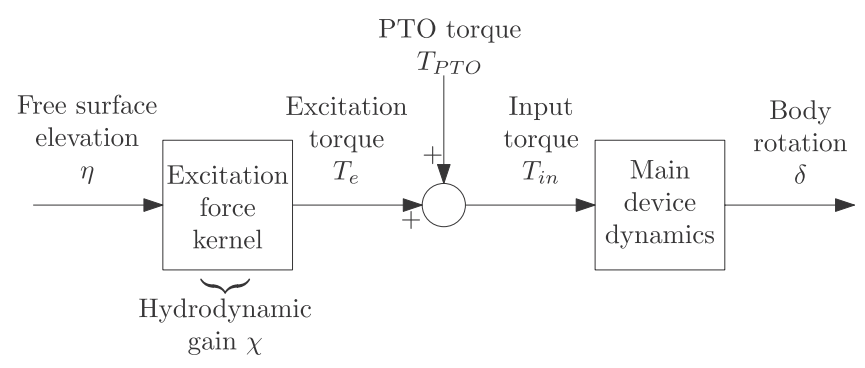

Fig. 1. General block diagram of the wave energy device model.

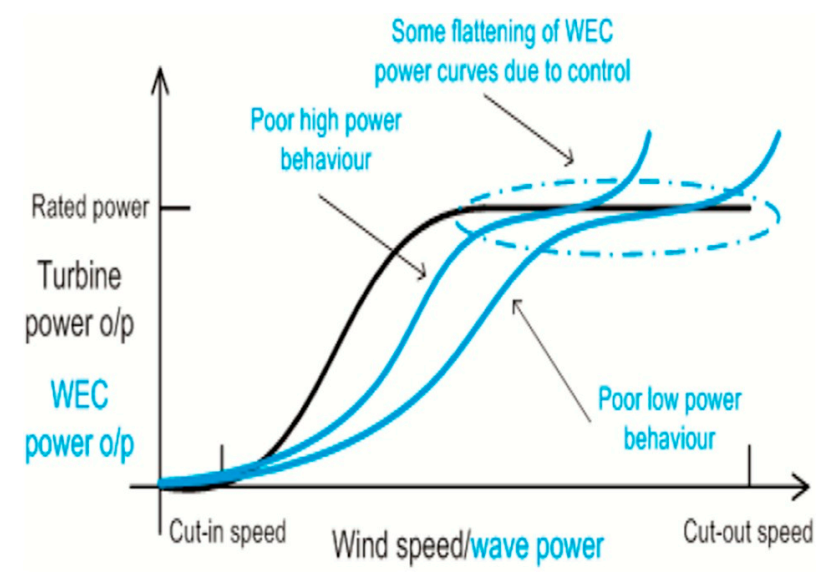

Fig. 2. Scheme of a $2 \mathrm{D}$ flat power curve, for wind turbine power and wave energy converter power.

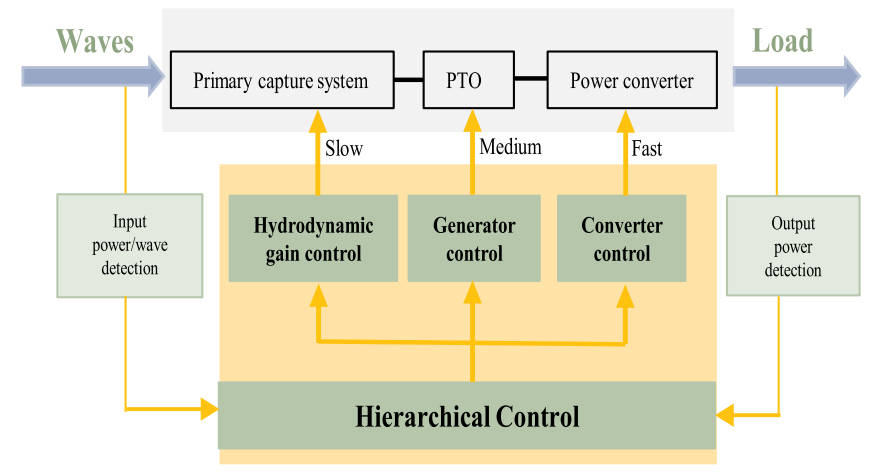

Fig. 3. Scheme of a hierarchical control system of a reconfigurable wave energy converter.

model variations across the full ranges of displacement and/or velocity of a classic flap. In contrast, our modelling approach will use linear hydrodynamics, but scheduled on the angle of four moveable vanes disposed on the flap. An appropriate mathematical model is vital for control and optimisation of WECs, since it is required for the assessment of forces and motion of devices and for use as a basis for model-based control design (Ringwood et al., 2014). To address the methodology of obtaining an appropriate parametric model, we follow the WEC concept and its geometry described in (Tom et al., 2016a). A drawing of the WEC is shown in Fig. 4. Variable geometries are obtained by adjusting the angles of the vanes, which are allowed to pitch about their center of rotation.

Previous studies on this WEC have shown that hydrodynamic parameters and time-average power are sensitive to vane angles, as indicated in (Tom et al., 2015), where a frequency domain analysis is carried out for the WEC when four vanes are actuated in synchronization (with

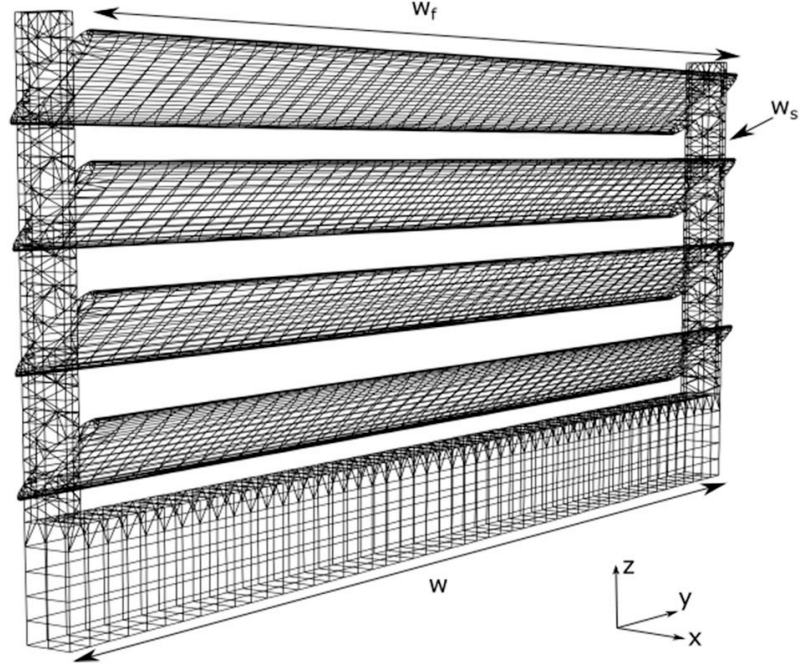

Fig. 4. $3 \mathrm{D}$ view of the mesh, for a vane angle $\theta=45^{\circ}$. Geometric parameters are given in Table 1.

an open angle of $0^{\circ}, 45^{\circ}$ or $90^{\circ}$ ). Subsequently, the performance of the WEC under irregular waves is investigated in (Tom et al., 2016b), with four vanes fully closed or fully opened. In (Tom et al., 2016a), the sensitivity of hydrodynamic coefficients and power performance, with respect to the thickness and width of the side and bottom support frame of this WEC, in regular waves, is investigated using a linear frequency domain analysis, assuming that the PTO system provides a constant and continuous linear damping coefficient. A pseudo-spectral method is used in (Tom et al., 2017a) and (Tom et al., 2017b) to balance the power-to-load ratio for the WEC with a non-ideal PTO when different numbers of vanes are fully opened or closed, under regular waves in (Tom et al., 2017a), and irregular waves in (Tom et al., 2017b).

The four identical elliptical vanes rotate about their center axis at the same time with the same angle $\theta$, measured positive clockwise from the radial axis of the body, as shown in Figs. 4 and 5. The geometric dimensions used can be found in Table 1 . The structural mass is evenly distributed and, as such, the pitch mass moment of inertia, I, and the linear hydrostatic restoring-torque coefficient $C_{h}$, will remain constant regardless of vane rotation. The structural mass density $\rho_{m}$ is set to half

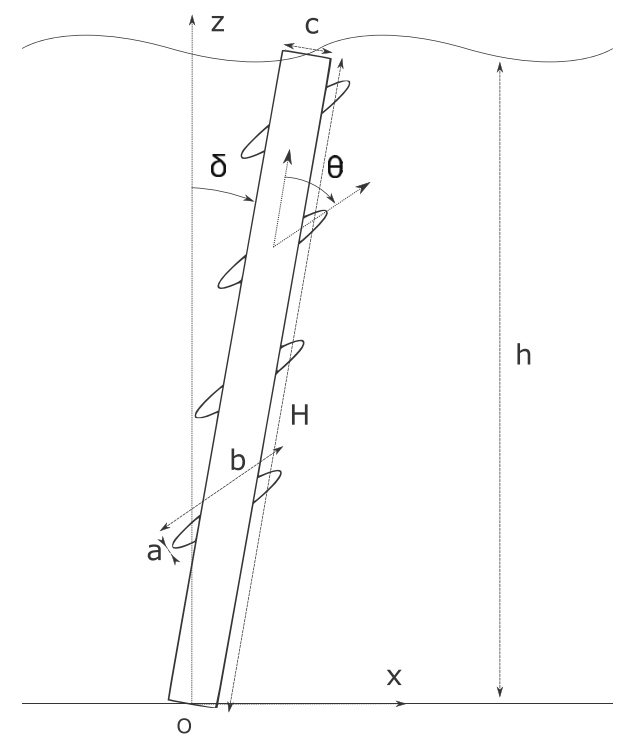

Fig. 5. Coordinate system for the device under investigation - schematic depicts the 2D $\theta=45^{\circ}$ geometry. Geometric parameters are given in Table 1 . 
Table 1

Geometric parameters for hydrodynamic modelling.

\begin{tabular}{llllll}
\hline Water depth & $h$ & $10 \mathrm{~m}$ & Vane minor axis & $a$ & $1 / 3 \mathrm{~m}$ \\
\hline WEC height & $H$ & $10 \mathrm{~m}$ & Vane major axis & $b$ & $2 \mathrm{~m}$ \\
WEC thickness & $c$ & $3 / 4 \mathrm{~m}$ & Side support width & $w_{s}$ & $1 / 4 \mathrm{~m}$ \\
WEC width & $w$ & $20 \mathrm{~m}$ & Center of gravity & $r_{g}$ & $3.97 \mathrm{~m}$ \\
Vane width & $w_{f}$ & $19.5 \mathrm{~m}$ & WEC mass & $m$ & $36 \mathrm{t}$ \\
WEC displaced & $V$ & $72 \mathrm{~m}^{3}$ & Mass moment of & $I$ & 923.4 \\
volume & & & inertia & & kg.m ${ }^{2}$ \\
\hline
\end{tabular}

the fluid density $\rho$.

In the current study, WAMIT Version 7 (WAMIT version 7.0, 2013). is used to compute the hydrodynamic parameters (added-mass, radiation damping, excitation force kernel) of the WEC, for frequencies from $\omega=0.1-3 \mathrm{rad} / \mathrm{s}$ at a spacing of $0.04 \mathrm{rad} / \mathrm{s}$. The vanes are controlled to move at the same time with the same angle $\theta$, varying from 0 to $90^{\circ}$.

Hydrodynamic parameters are computed for a set $\tilde{\theta}=$ $\left[0,1,4,9,25,40,50,59,70,80,90^{\circ}\right]$, containing a finite number of values of $\theta$. Those values have been chosen to be uniformly distributed over the complete range of $\theta$. However, closer values are picked for small angles of $\theta$, since hydrodynamic properties vary significantly in this range. Results are then interpolated to continuously describe the behaviour of the WEC device for the complete range of $\theta$ and wave frequencies $\omega$.

\section{Hydrodynamic model development}

\subsection{Frequency-domain formulation}

The WEC considered in the current article has one degree of freedom (1-DoF). The linearised equation of motion for a 1-DoF device can be expressed in the time domain in terms of Newton's second law, obtaining the following linear hydrodynamic formulation

$\left(I+\mu_{\infty}\right) \ddot{\delta}(t)+k_{r}(t) * \dot{\delta}(t)+B_{P T O} \dot{\delta}(t)+C_{h} \delta(t)=T_{e}(t)$,

where $I$ is the mass moment of inertia of the WEC, $\delta(t)$ the device pitch angle, $T_{e}(t)$ the wave excitation torque, $C_{h}>0$ the hydrostatic restoringtorque coefficient, $\mu_{\infty}=\lim _{\omega \rightarrow+\infty} A(\omega), \mu_{\infty}>0$ represents the addedmass $A(\omega)$ at infinite frequency, and $k_{r}(t) \in L^{2}(\mathbb{R})$ is the (causal) radiation impulse response, containing all the memory effect of the fluid response, and where $*$ represents a convolution product between $k_{r}(t)$ and the pitch speed $\dot{\delta}(t)$. $B_{\text {РтО }}$ is the power take-off (PTO) damping coefficient: a default linear damper PTO is employed here, since the focus of this study is hydrodynamic model, rather than control, set at $B_{\text {РTO }}=$ $3.10^{7}$ N.m.s.rad ${ }^{-1}$.

Since the mapping in (1) has a well-defined steady-state response, it is often useful to perform a frequency-domain analysis of such a system. Applying the Fourier transform to (1), and considering velocity as the measured output, the following representation holds

$\dot{\delta}(j \omega, \theta)=T_{e}(j \omega, \theta) H(j \omega, \theta)$,

where the excitation torque $T_{e}(j \omega, \theta)$ can be modelled as the free surface elevation $\eta(\omega)$ times the excitation force kernel $K(j \omega, \theta)$

$T_{e}(j \omega, \theta)=K(j \omega, \theta) \eta(\omega)$,

and where $H(j \omega, \theta)$ represents the force-to-velocity frequency response. $H(j \omega, \theta)$ is a function of a specific set of characteristic frequencydependent parameters, namely

$H(j \omega, \theta)=\frac{1}{B(\omega, \theta)+j \omega[A(\omega, \theta)+I]+B_{P T O}+\frac{C_{h}}{j \omega}}$,

where $B(\omega, \theta)$ is the radiation damping, and $A(\omega, \theta)$ the added-mass of the device (Falnes, 2002). The excitation force kernel $K(j \omega, \theta)$ and hydrodynamic parameters $A(\omega, \theta), B(\omega, \theta)$ can be efficiently obtained using existing BEM solvers, such as WAMIT (WAMIT version 7.0, 2013) or NEMOH (Babarit and Delhommeau, 2015).

\subsection{Ogilvie's relations: mapping between time and frequency}

Ogilvie (Ogilvie, Sep. 1964) established a direct relationship between time-domain (1) and frequency-domain (2) models, as a function of the parameters $B(\omega, \theta)$ and $A(\omega, \theta)$, and the radiation kernel $k_{r}(t)$, using the definition of the Fourier transform, namely

$B(\omega, \theta)=\int_{0}^{+\infty} k_{r}(t, \theta) \cos (\omega t) d t$,

$A(\omega, \theta)=\mu_{\infty}-\frac{1}{\omega} \int_{0}^{+\infty} k_{r}(t, \theta) \sin (\omega t) d t$.

It follows that the impulse response $k_{r}(t)$ can be written as a mapping involving the frequency-dependent parameters as

$k_{r}(t, \theta)=\frac{2}{\pi} \int_{0}^{+\infty} B(\omega, \theta) \sin (\omega t) d \omega$.

Equation (7) allows a frequency-domain analysis of $k_{r}(t)$; direct application of the Fourier transform yields

$k_{r}(j \omega, \theta)=B(\omega, \theta)+j \omega\left[A(\omega, \theta)-\mu_{\infty}\right] \equiv G(j \omega, \theta)$,

which leads to a frequency-domain description for $A(\omega, \theta)$ and $B(\omega, \theta)$, considering the real and imaginary parts, $\Re(G(j \omega, \theta))$ and $\Im(G(j \omega, \theta))$

$B(\omega, \theta)=\Re(G(j \omega, \theta))$,

$A(\omega, \theta)=\frac{1}{\omega} \Im(G(j \omega, \theta))+\mu_{\infty}$.

Obtaining a thorough description of the model requires finding a parametric modelling for the excitation force kernel $K(j \omega, \theta)(3)$ and radiation kernel $G(j \omega, \theta)(8)$, where both can be easily written as zeropole-gain transfer functions. The purpose of Sections 3 and 4 is to find a good parametrization for $K(j \omega, \theta)$ and $G(j \omega, \theta)$, for the complete range of $\theta$ and wave frequencies.

\subsection{Mesh generation and convergence study}

The geometry and mesh of the WEC device are created with Rhino Version 5. For a given sea-state, the geometry is fixed by a single angle $\theta$; therefore, the mesh is considered as a single body. Each mesh corresponds to one position of $\theta$.

A convergence study is achieved to set the number of panels required for hydrodynamic parameter computation in WAMIT. Two angles will be considered in this convergence study: $\theta=45^{\circ}$ and $\theta=90^{\circ}$.

The criteria for this convergence study is the relative error $\varepsilon$ on the maximum value of the radiation damping $B$, added-mass $A$ and excitation force kernel $K$ for the considered range of frequencies, given by the following equation

$\varepsilon_{X_{i}}=100\left|\frac{\max \left(X_{r e f}\right)-\max \left(X_{i}\right)}{\max \left(X_{r e f}\right)}\right|$,

where $X_{i}$ is, alternatively, the added mass $A$, the radiation damping $B$ or the excitation force kernel $K$ for a number $i$ of panels, and $X_{\text {ref }}$ are data from the reference mesh.

The reference mesh in both cases is the mesh with the highest number of panels studied, composed of about 16000 panels for both $\theta=$ $45^{\circ}$ and $\theta=90^{\circ}$.

Fig. $6 \mathrm{a}, \mathrm{b}$ and $6 \mathrm{c}$ show, in each case, that the relative error for all criteria is under $0.5 \%$ for meshes with more than 7000 panels; therefore, it will be the panel reference number for the following analysis. 


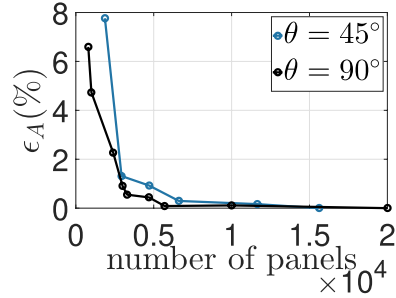

(a) Relative error on radiation added-mass $A$

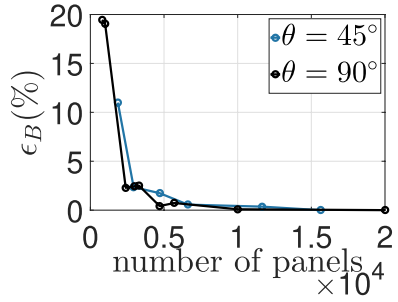

(b) Relative error on radiation damping $B$.

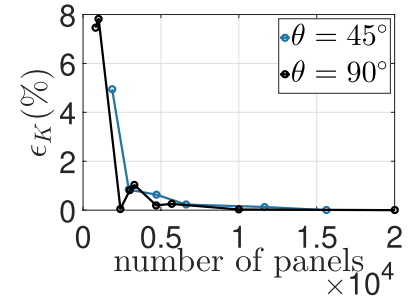

(c) Relative error on excitation force kernel $K$.

Fig. 6. Mesh convergence analysis for two configurations of vane angle, $\theta=45^{\circ}$ and $\theta=90^{\circ}$.

\section{Excitation force kernel modelling}

\subsection{Finite order parametrization of $K(j \omega, \theta)$ for a specific $\theta$}

Finding a parametrization of $K(j \omega, \theta)$ requires finding a finite order transfer function that fits well with WAMIT data over the range of wave frequencies, for any $\theta$. Different orders of transfer functions will be examined, from $N=2$ to $N=6$.

\subsubsection{Non causality of excitation force kernel}

The relationship between the free surface elevation $\eta$ and the excitation torque $T_{e}$ is non-causal (Falnes, 1995), which is also an issue for the identification. In our case trying to identify a non-causal system leads to an unstable one. There is therefore a necessity to split the system into a causal and a non-causal term before starting the identification process. For this purpose a pure advance $\tau$ is applied to the system

$K(j \omega)=e^{j \omega \tau} \tilde{K}(j \omega)$,

where $\tilde{K}(j \omega)$ is the causal excitation force kernel.

Before determining the optimal order $N$ for $\tilde{K}(j \omega), \tau$ has to be estimated. For this purpose, $N=6$ order systems are identified for different values of $\tau$, for the set of angles $\tilde{\theta}$, using a state-space model, identified using a subspace method (Overschee and Moor, 2012). For $\tau=1.745 \mathrm{~s}$ stable systems can be identified for the complete set of angles $\tilde{\theta}$.

\subsubsection{Optimal order of $\tilde{K}(j \omega)$}

For any order $N, \tilde{K}(j \omega)$ is given by the following equation.

$\tilde{K}(j \omega)=\frac{\chi}{\left(1+p_{1} j \omega\right)} \prod_{n=2}^{N} \frac{\left(1+z_{n} j \omega\right)}{\left(1+p_{n} j \omega\right)}$,

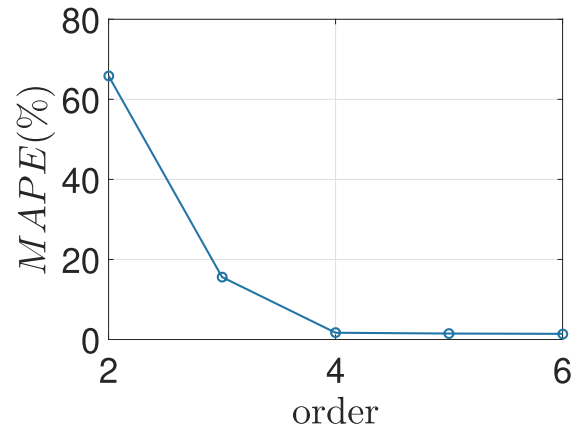

(a) MAPE of $K$, for orders from $N=2$ to $N=6$, for $\theta=45^{\circ}$. where $\chi$ is the hydrodynamic dc gain, $z_{n}$ are the zeros and $p_{n}$ the poles of $\tilde{K}(j \omega)$. The poles and/or zeros can be real or complex conjugate pairs. To find the optimal order $N$ for $\tilde{K}(j \omega, \theta)$, different order transfer functions are compared for a configuration of $\theta=45^{\circ}$.

The hydrodynamic gain $\chi$, the poles $p_{n}$ and the zeros $z_{n}$ of $\tilde{K}(j \omega)$ of order $N$ are obtained for each $\theta$ by minimization of an error function, given in terms of the Mean Absolute Percentage Error (MAPE) between the frequency domain data computed using the data from WAMIT $K_{W} \in$ $\mathbb{C}^{S \times 1}$ and the model $K \in \mathbb{C}^{S \times 1}$. The MAPE is given by

$\operatorname{MAPE}_{(K)}=\frac{100}{S} \sum_{i=1}^{S} \frac{\left|K_{W}\left(j \omega_{i}\right)-K\left(j \omega_{i}\right)\right|}{\left|K_{W}\left(j \omega_{i}\right)\right|}$.

Equation (13) is minimized with respect to $\chi, z_{1}, . ., z_{n}, . ., z_{N}, p_{1}, . ., p_{n}$, ..,$p_{N}$ using a sequential quadratic programming (SQP) algorithm (Nocedal and Wright, 1999). The MAPE is around $2 \%$ for $4^{\text {th }}$ order and greater, as shown in Fig. 7a, therefore order $N=4$ is selected for $\tilde{K}(j \omega)$. The MAPE is then computed for the complete range of $\theta$ in Fig. 7b, and gives a maximum error of $6.3 \% . \tilde{K}(j \omega)$ is a 8-parameter model, given by the following equation. where $\chi$ and $z_{1}$ are real numbers, while $z_{2}, p_{1}$ and $p_{2}$ are complex numbers, and $z_{2}^{*}, p_{1}^{*}$ and $p_{2}^{*}$ represent the complex conjugates of $z_{2}, p_{1}$ and $p_{2}$, respectively.

\subsection{Fitting functions of $\theta$ for $\tilde{K^{\sim}}(j \omega, \theta)$}

Each parameter of $\tilde{K}$ varies with $\theta$. However, since the variations are smooth, it is possible to find interpolation functions using rational functions for each parameter $p_{n}$ and $z_{n}$, as shown in Fig. 8. The gain $\chi$ is interpolated between data points using cubic interpolation. The fitting method for $\chi$ is chosen to reduce the MAPE, because of its significant

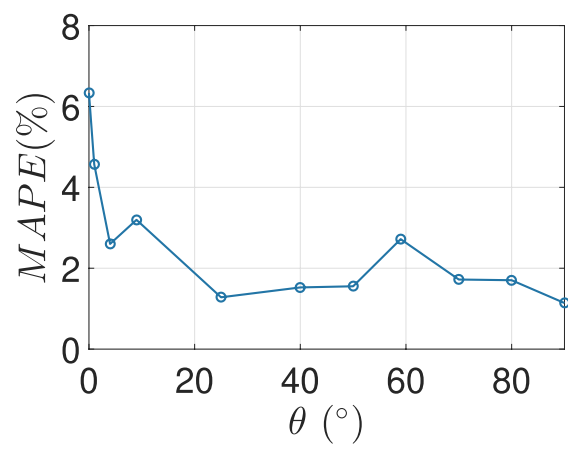

(b) MAPE of $K$ for chosen order $N=4$.

Fig. 7. Order analysis for $K$. Different orders of transfer function are anaylyzed in term of MAPE, for the $\theta=45^{\circ}$ configuration (left). Then, once the order chosen, the MAPE is studied on the complete range of $\theta$ (right).

$\tilde{K}(j \omega)=\frac{\chi j \omega\left(1+z_{1} j \omega\right)\left[1+z_{2} j \omega\right]\left[1+z_{2}^{*} j \omega\right]}{\left[1+p_{1} j \omega\right]\left[1+p_{1}^{*} j \omega\right]\left[1+p_{2} j \omega\right]\left[1+p_{2}^{*} j \omega\right]}$, 

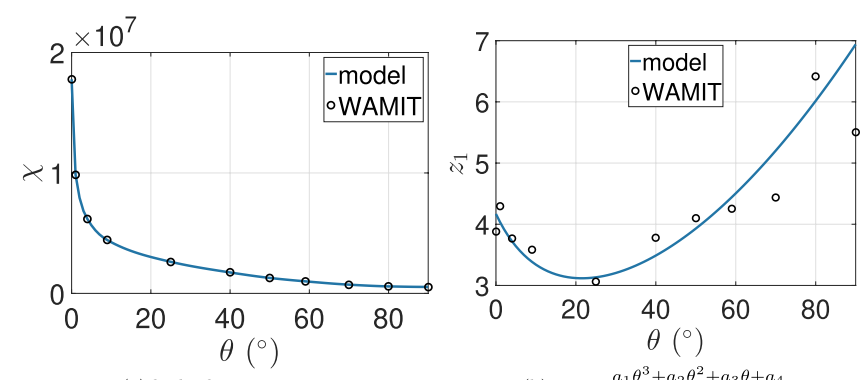

(a) hydrodynamic gain $\chi$
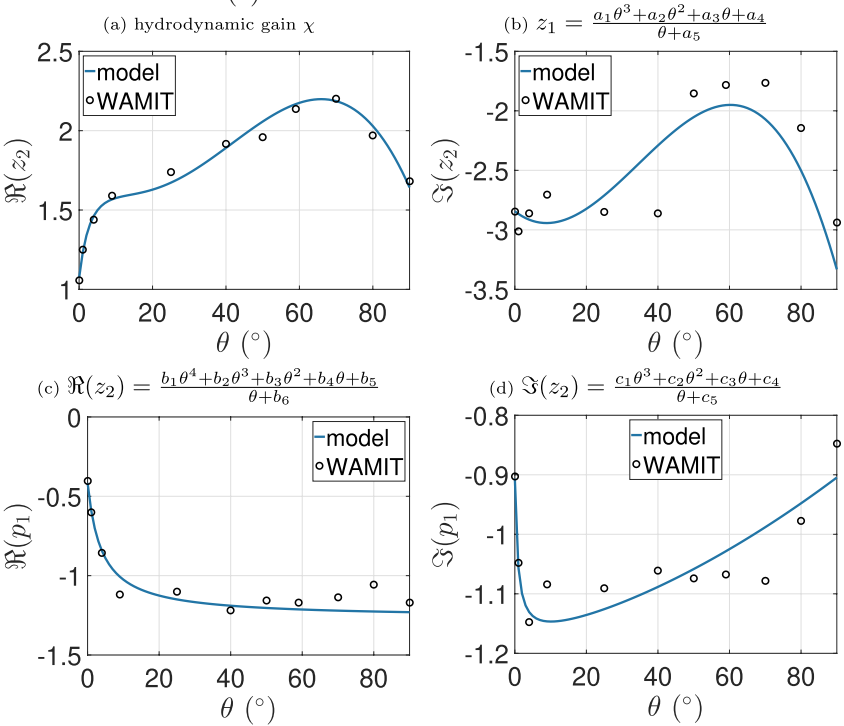

(d) $\Im\left(z_{2}\right)=\frac{c_{1} \theta^{3}+c_{2} \theta^{2}+c_{3} \theta+c_{4}}{\theta+c_{5}}$

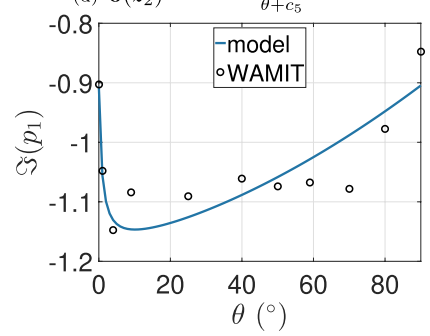

(e) $\Re\left(p_{1}\right)=\frac{d_{1} \theta+d_{2}}{\theta+d_{3}}$

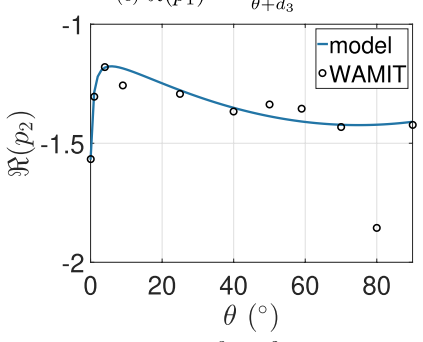

(g) $\Re\left(p_{2}\right)=\frac{f_{1} \theta^{3}+f_{2} \theta^{2}+f_{3} \theta+f_{4}}{\theta+f_{5}}$

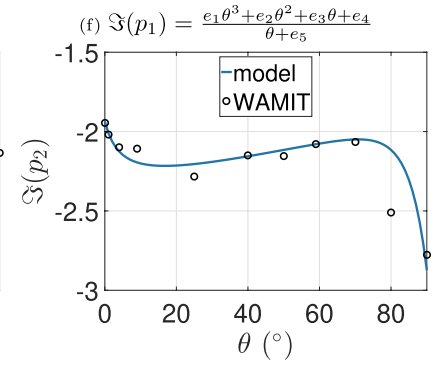

(h) $\Im\left(p_{2}\right)=\frac{g_{1} \theta^{2}+g_{2} \theta+g_{3}}{\theta+g_{4}}$

Fig. 8. Interpolated gain $\chi$, fitted poles $p_{n}$ and zeros $z_{n}$ for $K$, with their analytical expression.

variation over the range of $\theta$; for example, the value of $\chi$ for $\theta=90^{\circ}$, is more than 30 times lower than the value of $\chi$ for $\theta=0^{\circ}$. The hydrodynamic gain $\chi$ is the most important parameter in the error since it represents the overall (frequency independent) gain of the model.

\subsection{Results on excitation force kernel model}

Interpolation performance results are divided into two parts: model performance on training data $\tilde{\theta}=\left[0,1,4,9,25,40,50,59,70,80,90^{\circ}\right]$, and model validation data with a new set of angles $\tilde{\theta}_{v}=$ $\left[2,6,14,35,55,75,85^{\circ}\right]$.

\subsubsection{Excitation force kernel model creation}

Tables 2 and 3 lists the $R^{2}$ for each fitting function of the poles and

Table 2

$R^{2}$ fitting functions for zeros $z_{n}$.

\begin{tabular}{llll}
\hline Parameter & $z_{1}$ & $\Re\left(z_{2}\right)$ & $\Im\left(z_{2}\right)$ \\
\hline$R^{2}$ & 0.821 & 0.991 & 0.550 \\
\hline
\end{tabular}

Table 3

$R^{2}$ fitting functions for poles $p_{n}$.

\begin{tabular}{lllll}
\hline Parameter & $\Re\left(p_{1}\right)$ & $\Im\left(p_{1}\right)$ & $\Re\left(p_{2}\right)$ & $\Im\left(p_{2}\right)$ \\
\hline$R^{2}$ & 0.958 & 0.842 & 0.847 & 0.943 \\
\hline
\end{tabular}

zeros shown in Fig. 8. The interpolating functions have a relatively good $R^{2}$, except for $\Im\left(z_{2}\right)$. However it doesn't affect significantly the quality of the model, as MAPE stays under $7 \%$ for the complete range of $\theta$, as shown in Fig. 11a.

Fig. 9 shows the behaviour of the magnitude and phase of $K$ for the complete range of frequencies and angles $\theta$. The sensitivity of the gain $\chi$ is reflected in the amplitude of $|K|$ in Fig. 10; when $\theta$ varies from 0 to $4^{\circ}$, amplitude decreases by a factor of 2 . Then, the sensitivity quickly falls off as $\theta$ grows.

\subsubsection{Excitation force kernel model validation}

The MAPE between WAMIT data and the model $K$ are compared for a validation set $\tilde{\theta}_{v}$, as shown in Fig. 11b. Even though the error is more significant for large $\theta$, the MAPE stays under 10\%. Fig. 11b also shows that the error increases as $\chi$ decreases, which leads that the greatest errors are made on the most negligible values of $\chi$.

\section{Radiation kernel modelling}

\subsection{Finite order parametrization of $G(j \omega, \theta)$ for a specific $\theta$}

As in Section 3, the same process is applied to find a parametrization for the radiation kernel model $G(j \omega)$, using orders from $M=2$ to $M=6$. For any order $M, G(j \omega)$ is given by the following equation

$G(j \omega)=\frac{\kappa}{\left(1+r_{1} j \omega\right)} \prod_{n=2}^{M} \frac{\left(1+q_{n} j \omega\right)}{\left(1+r_{n} j \omega\right)}$,

where $\kappa$ is the dc gain. $q_{n}$ are the zeros and $r_{n}$ the poles of $G(j \omega)$, where the poles and/or zeros can be real or complex conjugate pairs. As for excitation force kernel modelling case, the best order for $G(j \omega, \theta)$ is identified for a configuration $\theta=45^{\circ}$.

The gain $\kappa$, the poles $r_{n}$ and the zeros $q_{n}$ of $G(j \omega)$ of order $M$ are obtained for each $\theta$ by minimization of the MAPE between the frequency domain data computed using the data from WAMIT $G_{W} \in \mathbb{C}^{S \times 1}$ and the model $G \in \mathbb{C}^{S \times 1}$

$\operatorname{MAPE}_{(G)}=\frac{100}{S} \sum_{i=1}^{S} \frac{\left|G_{W}\left(j \omega_{i}\right)-G\left(j \omega_{i}\right)\right|}{\left|G_{W}\left(j \omega_{i}\right)\right|}$.

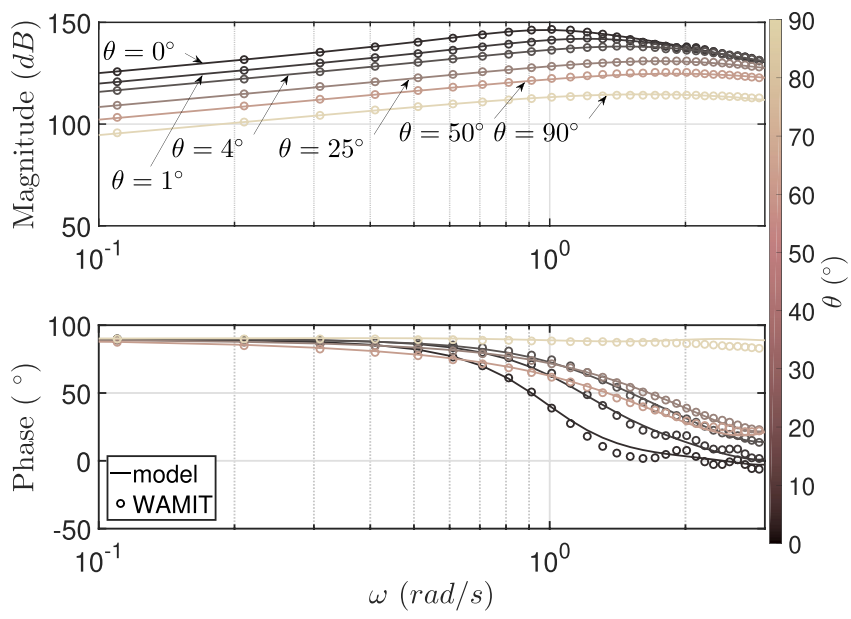

Fig. 9. Magnitude and phase of excitation force kernel K. 


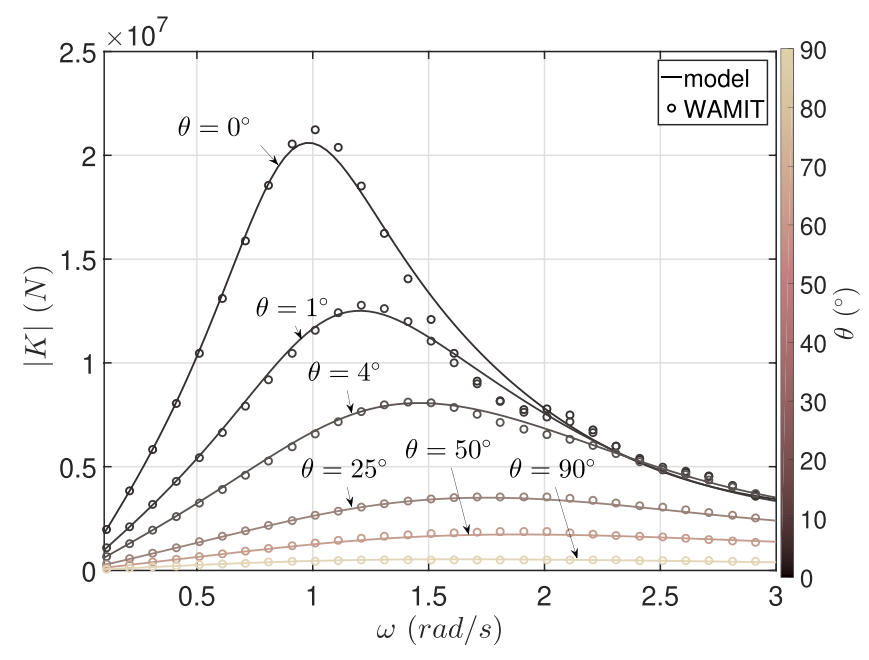

Fig. 10. Excitation force kernel amplitude $|K|$.

Equation (16) is minimized with respect to $\kappa, q_{1}, . ., q_{n}, . ., q_{M}, r_{1}, . ., r_{n}$, .., $r_{M}$ using a SQP algorithm. The MAPE is around $2 \%$ for orders $M=5$ and larger, as shown in Fig. 12a, therefore, order $M=5$ is selected. The MAPE is then computed for the complete range of $\theta$ in Fig. 7b, and gives a maximum error of $2.5 \% . G(j \omega)$ is a 6-parameter model given by the following equationwhere $\kappa, q_{1}, q_{2}, r_{1}$ and $r_{3}$ are real, $r_{2}$ is a complex number and $r_{2}^{*}$ its conjugate. $r_{3}$ is a pole fixed at $r_{3}=30$.

\subsection{Fitting functions of $\theta$ for $G(j \omega, \theta)$}

The poles $r_{n}$ and zeros $q_{n}$ are interpolated using arc tangent, exponential and rational functions, as shown in Fig. 13. The gain $\kappa$ is fitted with cubic interpolation because of its significant variation over the range of $\theta$; for $\theta>60^{\circ}, \kappa$ is more than 100 times lower than the value of $\kappa$ for $\theta=0^{\circ}$. We can notice that the hydrodynamic gain $\chi$ obtained in Fig. 8a has a direct impact on the radiation kernel gain $\kappa$ behaviour in Fig. 13a.

\subsection{Added-mass at infinite frequency $\mu_{\infty}$}

To obtain the added-mass from $G$, the added mass at infinite frequency term $\mu_{\infty}$ is required in Equation (9). As for the gain $\kappa, \mu_{\infty}$ is obtained from WAMIT and interpolated between data points using cubic interpolation, as shown in Fig. 14.

\subsection{Results on radiation kernel model}

As for the excitation force kernel model in Section 3.3, the radiation kernel model results are divided into performance on training data $\tilde{\theta}$, and validation on $\tilde{\theta}_{v}$.

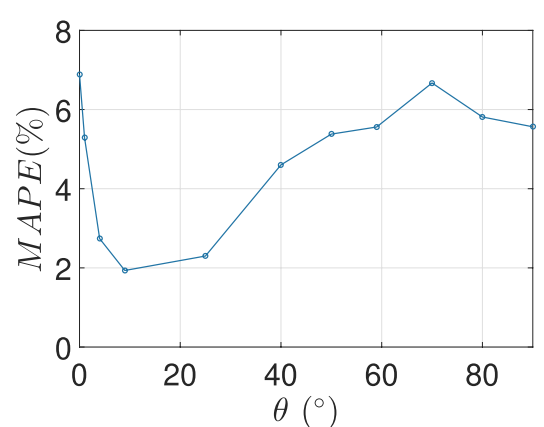

(a) MAPE of $K$ for model creation.

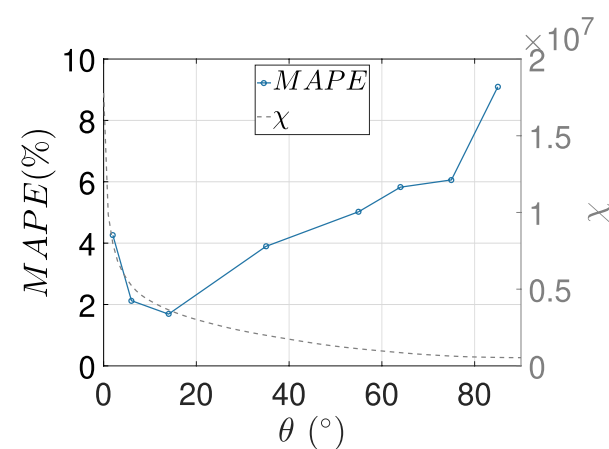

(b) MAPE of $K$ for model validation (in solid lines) - hydrodynamic gain $\chi$ (in dashed lines).

Fig. 11. MAPE of excitation force kernel on $\tilde{\theta}$, used for the creation of the model $K$ (on the left), and MAPE of excitation force kernel on $\tilde{\theta}_{v}$, used for validation (on the right).

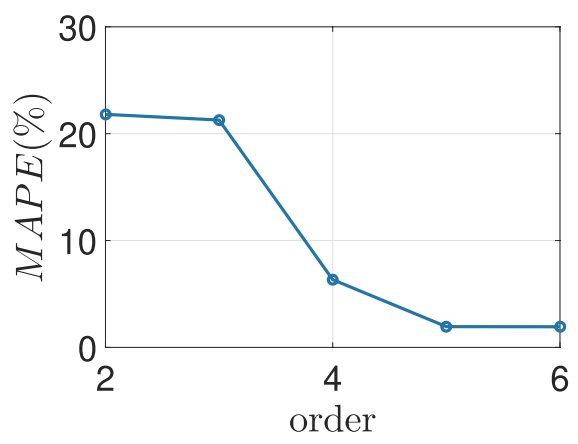

(a) MAPE of $G$, for orders from $M=2$ to $M=6$, for $\theta=45^{\circ}$

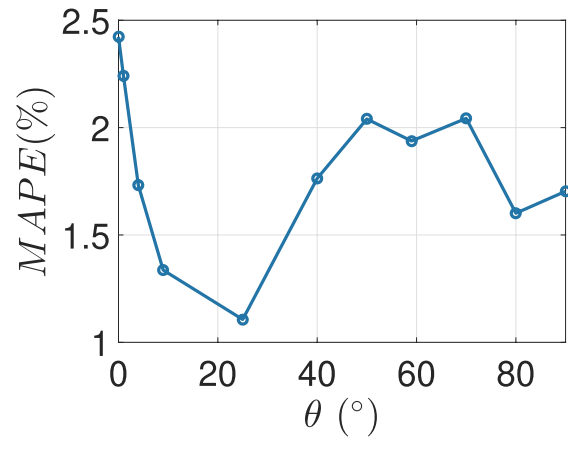

(b) MAPE of $G$ for chosen order $M=5$.

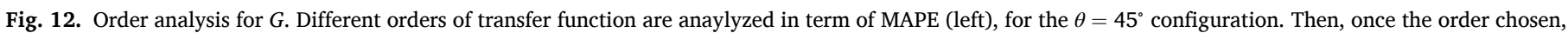
the MAPE is studied on the complete range of $\theta$ (right).

$G(j \omega)=\frac{\kappa j \omega\left(1+q_{1} j \omega\right)\left(1+q_{2} j \omega\right)^{2}}{\left(1+r_{1} j \omega\right)^{2}\left[1+r_{2} j \omega\right]\left[1+r_{2}^{*} j \omega\right]\left(1+r_{3} j \omega\right)}$, 

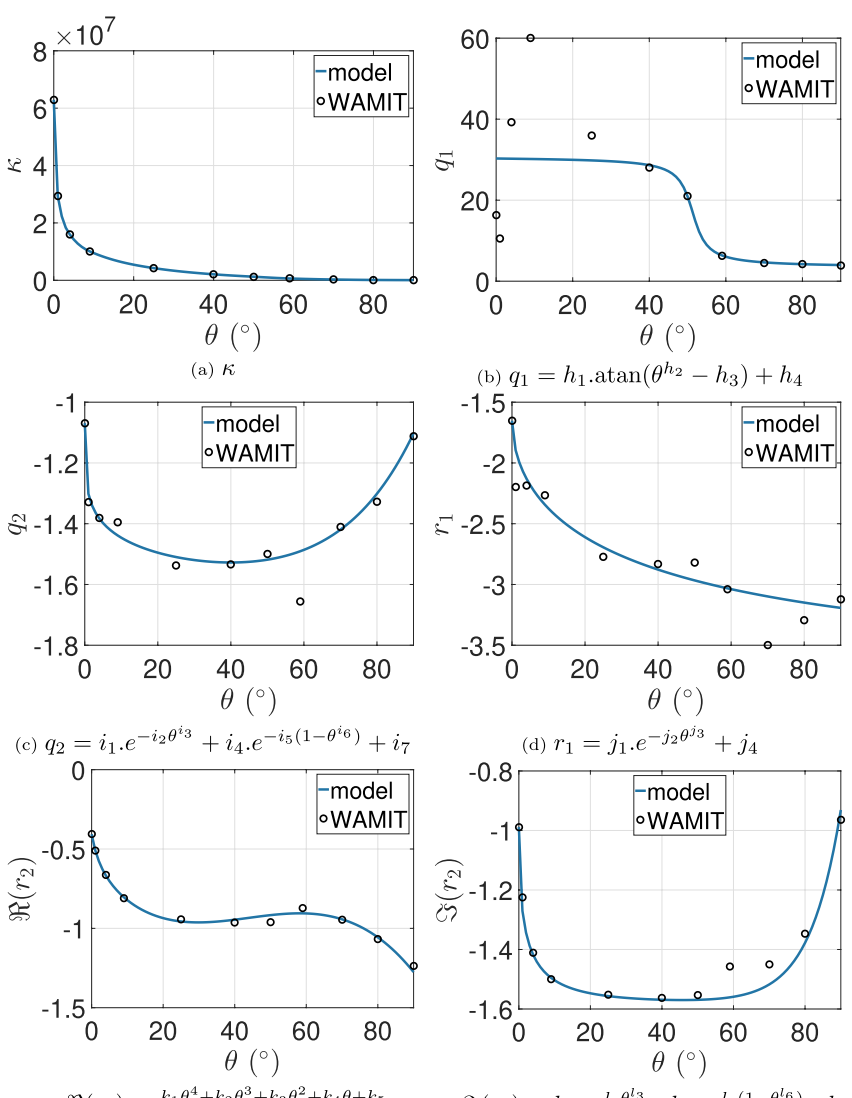

(e) $\Re\left(r_{2}\right)=\frac{k_{1} \theta^{4}+k_{2} \theta^{3}+k_{3} \theta^{2}+k_{4} \theta+k_{5}}{\theta+k_{6}}$

(f) $\Im\left(r_{2}\right)=l_{1} \cdot e^{-l_{2} \theta^{l_{3}}}+l_{4} \cdot e^{-l_{5}\left(1-\theta^{l_{6}}\right)}+l_{7}$

Fig. 13. Interpolated gain $\kappa$, fitted poles $r_{n}$ and zeros $q_{n}$ for $G$, with their analytical expression.

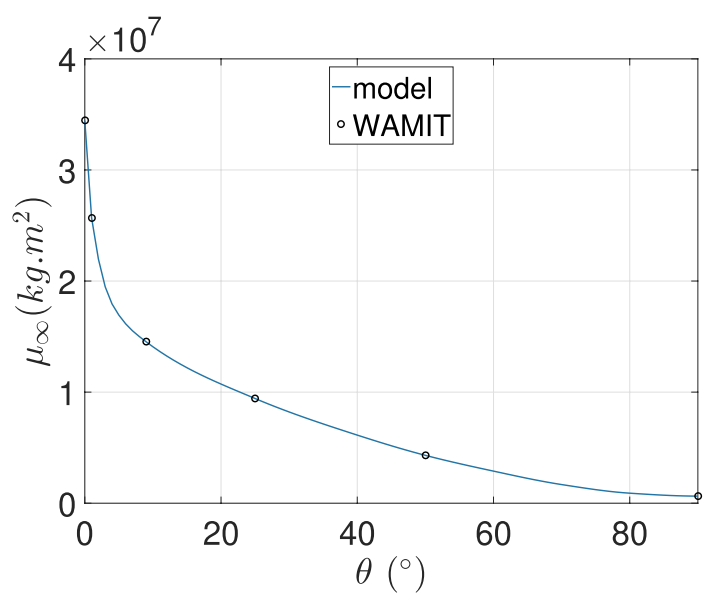

Fig. 14. Added-mass at infinite frequency $\mu_{\infty}$.

\subsubsection{Radiation kernel model creation}

Table 4 lists the $R^{2}$ values for each fitting function for the poles $r_{n}$ and zeros $q_{n}$ shown in Fig. 13. Fitting functions have relatively good $R^{2}$ values, except $q_{1}$. However, it has a very negligible impact on the MAPE, since large values of poles or zeros affect only high frequency behaviour,

Table 4

$R^{2}$ fitting functions for poles $r_{n}$ and zeros $q_{n}$.

\begin{tabular}{llllll}
\hline Parameter & $q_{1}$ & $q_{2}$ & $r_{1}$ & $\Re\left(r_{2}\right)$ & $\Im\left(r_{2}\right)$ \\
\hline$R^{2}$ & 0.520 & 0.898 & 0.898 & 0.965 & 0.958 \\
\hline
\end{tabular}

which is beyond the studied frequency range.

Fig. 15 shows the behaviour of $G$ for the complete range of wave frequencies and $\theta$. The MAPE for $G$ is shown, for each $\theta$, in Fig. 18a.

The MAPE stays under 7\% for the complete range of $\theta$. Figs. 16 and 17 show that, for a variation from $\theta=0^{\circ}$ to $\theta=1^{\circ}$, both added-mass and radiation damping almost decrease by a factor of 2 . This is explained by the gain $\kappa$ in Fig. 13, which is very sensitive for small $\theta$, and decreases as $\theta$ increases.

\subsubsection{Radiation kernel model validation}

As in Section 3.3.2, WAMIT data are compared to the model $G$ with the validation set $\tilde{\theta}_{v}$ using the MAPE, as shown in Fig. 18b. Even though the error is more significant for large $\theta$, the MAPE stays under $11 \%$. Fig. 18b also shows that the error increases as $\kappa$ decreases, which leads that the greatest errors are made on the most negligible values of $\kappa$.

\section{Application example: power curve calculation in regular waves}

The purpose here is to use the models defined in Sections 3 and 4 to analyse the behaviour of $\theta$ depending on frequency and amplitude of the waves in regular waves, when trying to maximize power absorption. In addition we take into account a rated power $P_{r}$, corresponding to the maximum power above which the device is set on survival mode. The rated power depends on the maximum instantaneous absorbed power, which itself depends on several parameters, such as the WEC geometry, power-take-off, load foundations, which is not subject to the current article. We consider a maximum instantaneous absorbed power for the WEC of $P_{\max }=500 \mathrm{~kW}$. Considering time-averaged power in regular waves, rated power $P_{r}$ is half of $P_{\max }$, giving $P_{r}=250 \mathrm{~kW}$.

The method used to determine the appropriate values for $\theta$ was to back solve from the rated power to determine the appropriate value for $\theta$. It is then possible to store these $\theta$ values in a look-up table, which could be indexed by sea state and/or wave power.

\subsection{Power equation}

From Equation (2), we can get the detailed equation of motion in the frequency domain

$\dot{\delta}(j \omega, \theta)=\frac{K(j \omega, \theta) \eta(\omega)}{B_{m}(\omega, \theta)+j \omega\left[A_{m}(\omega, \theta)+I\right]+B_{P T O}+\frac{C_{h}}{j \omega}}$.

The wave power for incident regular waves in finite depth is given by the following equation

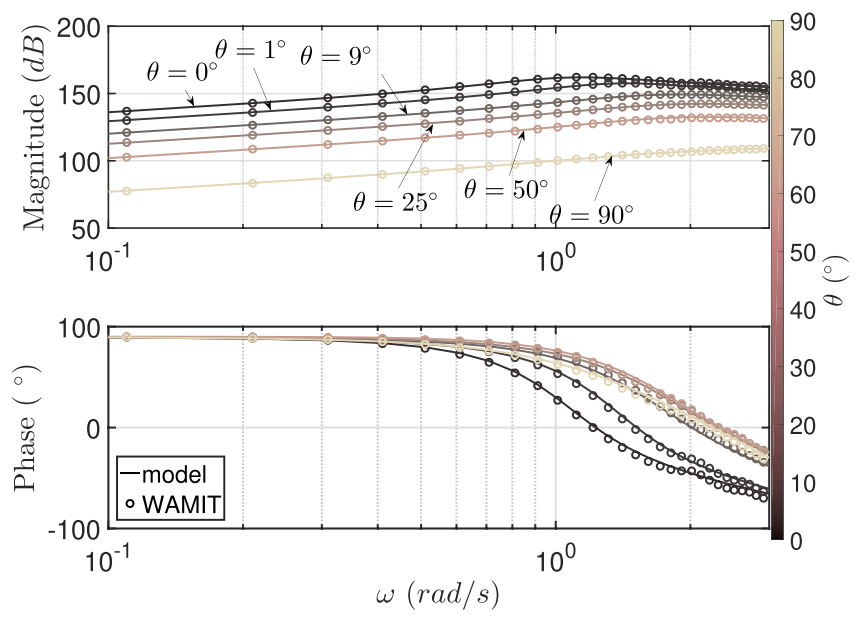

Fig. 15. Magnitude and phase of radiation kernel G. 


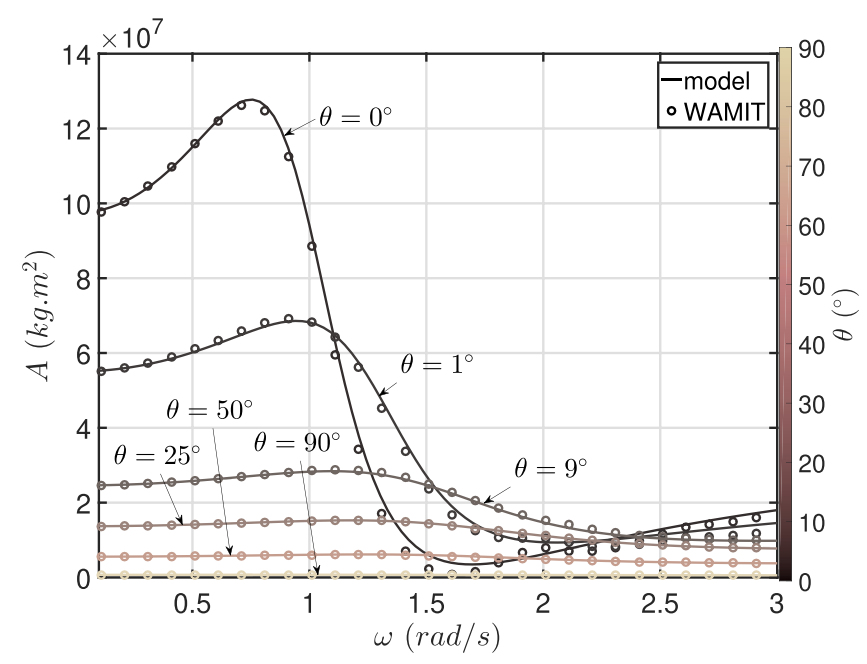

Fig. 16. Added-mass A obtained from radiation kernel model G.

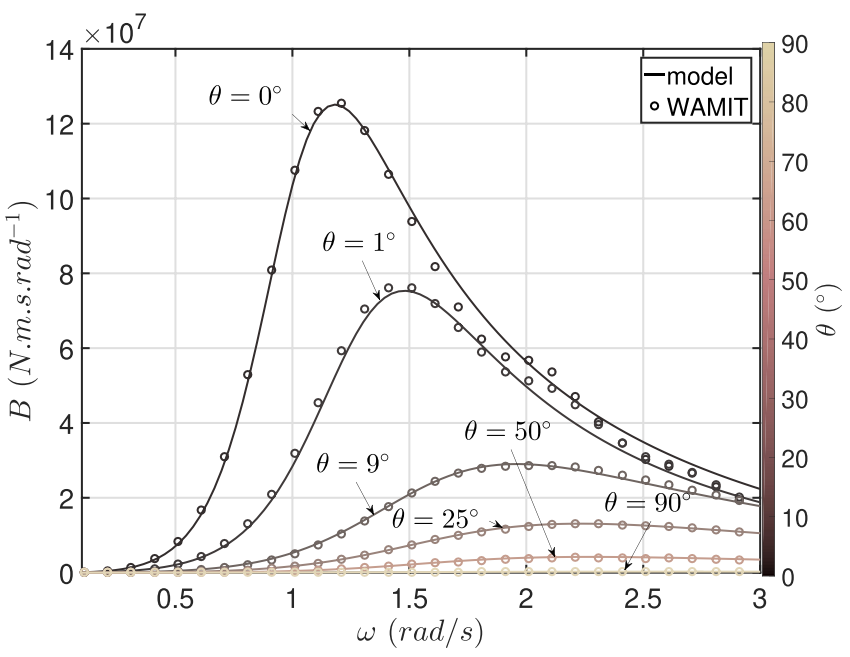

Fig. 17. Radiation Damping B obtained from radiation kernel model G.

$P_{w}=\frac{w \rho g^{2} a_{w}^{2}}{8} \sqrt{\frac{g}{k} \tanh (k h)}$,

with the relation dispersion

$\omega^{2}=g k \tanh (k h)$,

where $\rho$ is the fluid density, $g$ the acceleration due to the gravity, $a_{w}$ is the wave amplitude, $w$ the width of the WEC, $h$ the water depth, and $k$ the wavenumber.

The averaged absorbed power $P_{a b s}$, considering a linear PTO damping $B_{\text {РТO }}$, is given by

$P_{a b s}=\frac{1}{2} B_{P T O}|\dot{\delta}|^{2}$.

\subsection{Power absorption with $\theta$ control (3D)}

Fig. 19 shows $P_{a b s}$ and $\theta$ as a function of the wave period $T$ and the amplitude $a_{w}$, taking into account the rated power $P_{r}$. For small values of $T$ and/or $a_{w}, P_{a b s}<P_{r}$, and $\theta$ stays at $0^{\circ}$, no control on $\theta$ is needed. When the maximum absorbed power $P_{a b s}$ reaches the rated power $P_{r}$, power is spilled by moving the vane angle $\theta$, in order to stay at the maximum power absorption value $P_{r}$. Fig. 20 shows the variation of $\theta$ which maximizes power absorption (taking into account $P_{r}$ ), and wave power. We can see that when the rated power is reached, $\theta$ increases as the input power $P_{w}$ increases. An interesting fact is that, for a given value of wave power, there is no unique position for $\theta$. With the given WEC geometry, absorbed power $P_{a b s}$ never exceeds rated power $P_{r}$, and the margin of spilled power is large, given that the maximum value of $\theta$ reaches only $60^{\circ}$. Fig. 21 shows the pitch angle $\delta$, using $\theta$ control. $\delta$ stays under $16^{\circ}$ for the complete range of amplitude and period.

\subsection{Power absorption with $\theta$ control (2D)}

To get a 2D flat power curve similar to that for a wind turbine shown in Fig. 2, the wave power, which depends on both amplitude and

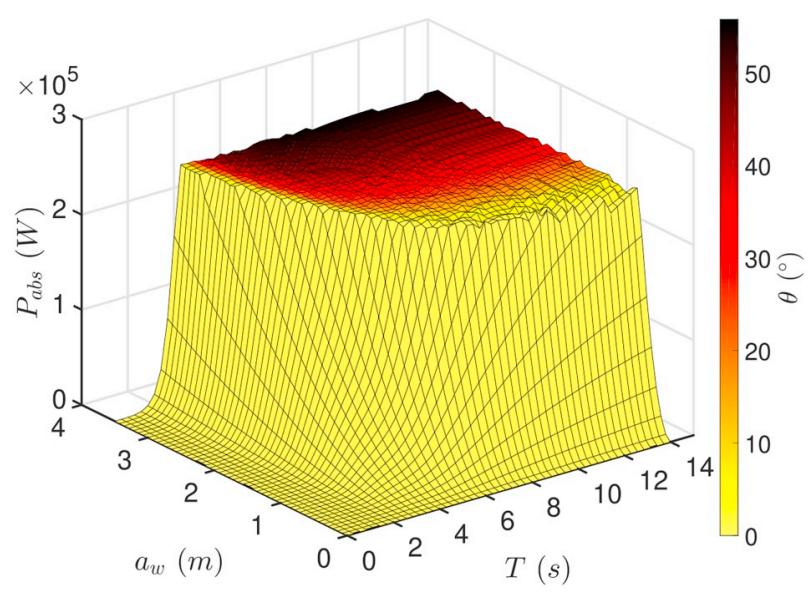

Fig. 19. $P_{a b s}$ and $\theta$ as a function of the wave amplitude $a_{w}$, and period T, using $\theta$ control.

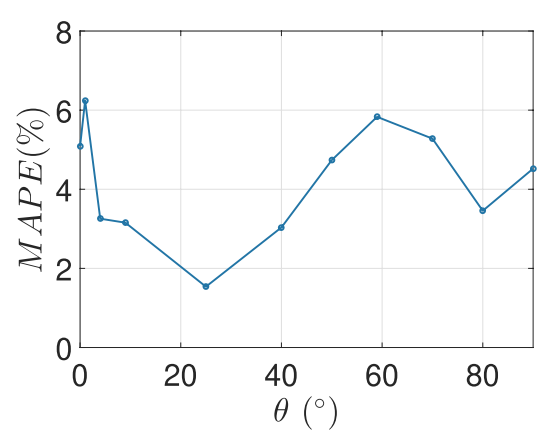

(a) MAPE of $G$ for model creation.

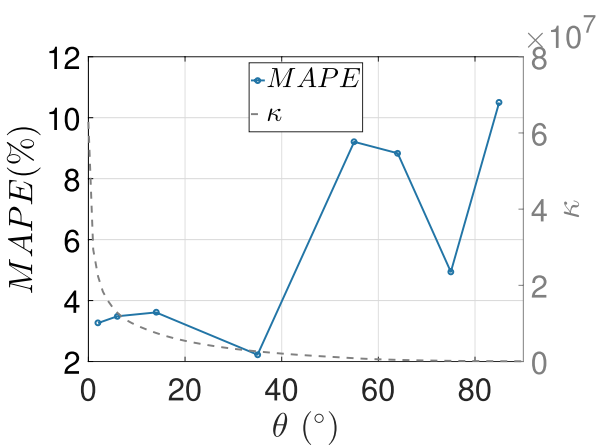

(b) MAPE of model $G$ for model validation (in solid lines) - gain $\kappa$ (in dashed lines.

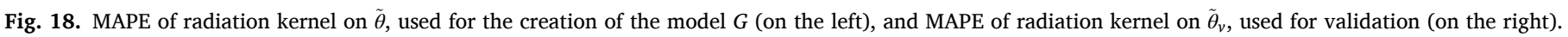




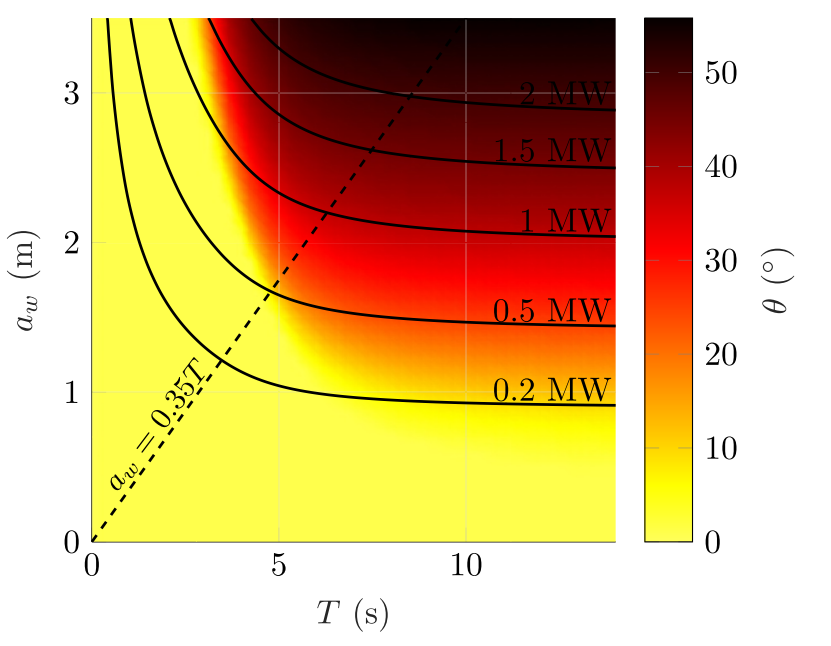

Fig. 20. Wave power $P_{w}$ and $\theta$ as a function of the wave amplitude $a_{w}$, and period $\mathrm{T}$, using $\theta$ control. The dashed line is used in Section 5.3 to obtain Fig. 22.

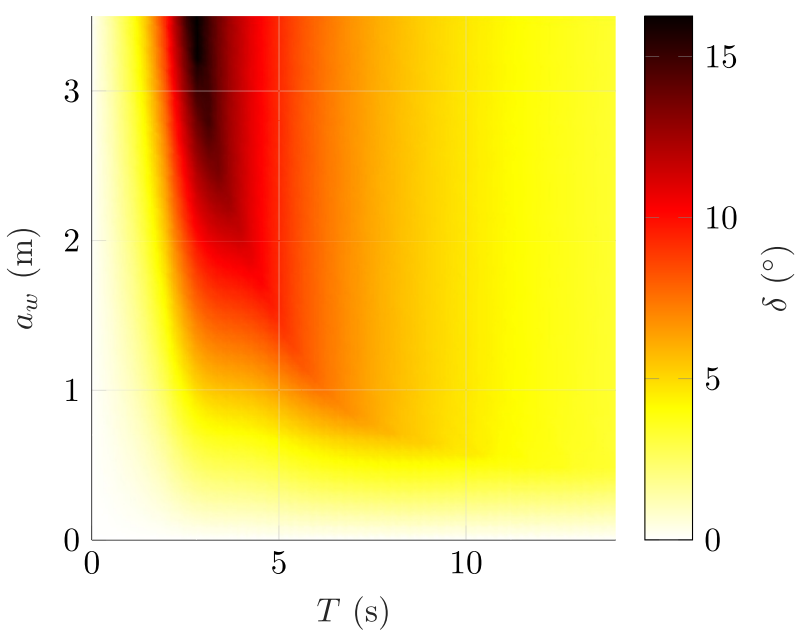

Fig. 21. Pitch angle $\delta$ as a function of the wave amplitude $a_{w}$, and period T, using $\theta$ control.

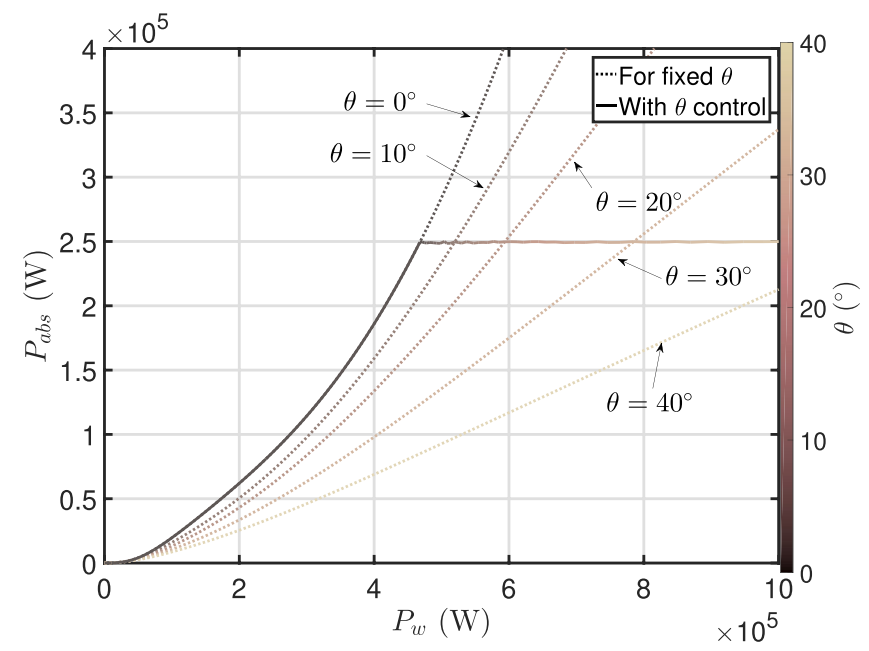

Fig. 22. Absorbed power without $\theta$ control (dashed lines), and with $\theta$ control (solid line). frequency has to be described in a 1D way. For this purpose, a link between amplitude $a_{w}$ and period $T$ is established as follows

$a_{w}=0.35 T$.

Equation (22) is not based on any theoretical relationship between $a_{w}$ and $T$, but rather shows a plausible relationship, garnered from the straight line relationship shown in Fig. 20. This allows a 2-D plot of output power, $P_{a b s}$, Vs input power, $P_{w}$, to be drawn, thus making the connection with typical wind turbine power curves, shown in Fig. 22. $P_{a b s}$ is shown for different values of $\theta$ without taking a limit power into account, and then with $\theta$ control, showing adherence to rated power.

Without $\theta$ control, Fig. 22 shows that for small values of $\theta$ the absorbed power quickly reaches $P_{r}$ as the wave power increases, which implies the device to be put in survivability mode from moderate seastates. In contrast, fixing a large value for $\theta$ permits a larger operating area, however the absorbed power stays widely below $P_{r}$. Applying $\theta$ control allows the WEC to spill power, which leads to a constant absorbed power equal to $P_{r}$, for any wave power.

\subsection{Discussion of results}

Fig. 22 shows that a wind-turbine-like power curve can be synthesised for a wave energy device. The curve shown in Fig. 22 assumes that the input power is constant, so that a fixed value of input power and vane angle $\theta$ gives a constant absorbed power. In reality, of course, the input power will be variable, within defined limits, for any sea state, due to the stochastic nature of irregular waves. In order to smooth absorbed power, a fast real-time controller (such as described in (Faedo et al., 2017)), continuously adjusting the opposing torque of the vane, $T_{\text {Рто, }}$ will be used in concert with an upper level controller used to vary the vane angle in response to (slower) sea state variations. This also requires some measure (or forecast) of the current, and/or future, sea state variations.

These are topics for future research. What the present article does show is that it's feasible to achieve a flat power curve and the model developed in Section 3 and 4 can be used in the design of both the seastate (actuating $\theta$ ) controller and the real-time (actuating $T_{\text {РтO) }}$ controller, as well as possible uses in simulation, optimisation, and power production assessment studies.

One question which might reasonably be asked is the extent to which the validity of our linear hydrodynamic model extends. Here, we can reference the work of McCabe et al. (2006), who show that there is insignificant departure between linear and nonlinear up to displacement angles of $20^{\circ}$. In our case, as is clear from Fig. 21, the displacement angle of the device does not exceed $16^{\circ}$, mainly by virtue of the ability to spill excitation force, therefore preventing large displacement excursions.

\section{Conclusion}

This paper presents a parametric model for the reconfigurable wave energy converter developed at the National Renewable Energy Laboratory in the US. The approach is based on multiple linear modelling, and the orders of the parametric models provide a trade-off between computation complexity and approximation accuracy. Numerical results show that the compact model developed can accurately model the hydrodynamics of the device, which makes it well suited to the modelbased control of wave energy converters.

The example case study shows that the parametric model can be used to modulate the power transfer from incident ocean wave to the power take-off system of wave energy converters. Using vane angle as a control variable, the hydrodynamic gain can be tuned and the ubiquitous flat power curve can be obtained for wave energy devices. This indicates a pragmatic approach to improve the devices' survivability in harsh sea and to increase the quality of generated power. 


\section{Acknowledgments}

This paper is based on upon work supported by Science Foundation Ireland under Grant No. SFI/13/IA/1886 BU:2600214.

\section{References}

Babarit, A., Delhommeau, G., Sep. 2015. Theoretical and numerical aspects of the open source BEM solver NEMOH. In: 11th European Wave and Tidal Energy Conference (EWTEC2015). Proceedings of the 11th European Wave and Tidal Energy Conference. Nantes, France.

Crooks, D.J., 2017. Nonlinear Hydrodynamic Modelling of an Oscillating Wave Surge Converter. Ph.D. thesis. Queen's University Belfast.

WAMIT version 7.0, 2013. User manual. http://www.wamit.com. (Accessed 17 October 2018).

Faedo, N., Olaya, S., Ringwood, J., Sep. 2017. Optimal control, MPC and MPC-like algorithms for wave energy systems: an overview. IFAC J. Syst. Contr. 1, 37-56.

Falcão, A., Apr, 2010. Wave energy utilization: a review of the technologies. Renew. Sustain. Energy Rev. 14 (3), 899-918.

Falnes, J., Dec. 1995. On non-causal impulse response functions related to propagating water waves. Appl. Ocean Res. 17 (6), 379-389.

Falnes, J., 2002. Ocean Waves and Oscillating Systems: Linear Interactions Including Wave-Energy Extraction. Cambridge University Press.

McCabe, A., Aggidis, G., Stallard, T., 2006. A time-varying parameter model of a body oscillating in pitch. Appl. Ocean Res. 28 (6), 359-370.

Murray-Smith, R., Johansen, T., 1997. Multiple Model Approaches to Nonlinear Modelling and Control. CRC press.
Nocedal, J., Wright, S.J., 1999. Numerical Optimization, Chapter 18. Springer Series in Operations Research. Springer, New York.

Ogilvie, T., Sep. 1964. Recent progress toward the understanding and prediction of ship motions. In: Fifth Symposium on Naval Hydrodynamics 1, pp. 2-5.

Overschee, P. v., Moor, B. L. d., Dec. 2012. Subspace Identification for Linear Systems: Theory - Implementation - Applications. Springer Science \& Business Media.

Ringwood, J.V., Simani, S., 2015. Overview of modelling and control strategies for wind turbines and wave energy devices: comparisons and contrasts. Annu. Rev. Contr. 40, $27-49$.

Ringwood, J., Bacelli, G., Fusco, F., 2014. Energy-maximizing control of wave-energy converters: the development of control system technology to optimize their operation. IEEE Control Syst. Mag. 34 (5), 30-55.

Tom, N., Lawson, M., Yu, Y., Wright, A., 2015. Preliminary analysis of an oscillating surge wave energy converter with controlled geometry. In: Proceedings of the 11th European Wave and Tidal Energy Conference. Sep.

Tom, N., Lawson, M., Yu, Y., Wright, A., 2016. Development of a nearshore oscillating surge wave energy converter with variable geometry. Renew. Energy 96, 410-424. Oct.

Tom, N., Lawson, M., Yu, Y., Wright, A., 2016. Spectral modeling of an oscillating surge wave energy converter with control surfaces. Appl. Ocean Res. 56, 143-156. Mar.

Tom, N., Yu, Y., Wright, A., 2017. Balancing the Power-To-Load Ratio for a Novel Variable Geometry Wave Energy Converter with Nonideal Power Take-Off in Regular Waves: Preprint. National Renewable Energy Lab. (NREL), Golden, CO (United States). Tech. Rep. NREL/CP-5000-68082.

Tom, N., Yu, Y., Wright, A., Lawson, M., 2017. Balancing power absorption against structural loads with viscous drag and power-takeoff efficiency considerations. IEEE J. Ocean. Eng. 1-20. 